\title{
Status de fragilidade entre idosos com indicativo de depressão segundo o sexo
}

\author{
Fragility status among elderly with indicative of depression by gender
}

Darlene Mara dos Santos Tavares', Esthefânia Garcia de Almeida', Pollyana Cristina dos Santos Ferreira', Flavia Aparecida Dias', Maycon Sousa Pegorari

\section{RESUMO}

Objetivos: Descrever as variáveis socioeconômicas de idosos com indicativo de depressão segundo o sexo, verificar a associação entre o status de fragilidade e o sexo, e descrever o componente do fenótipo de fragilidade mais impactado entre os idosos com indicativo de depressão pré-frágeis e frágeis. Métodos: Estudo observacional, transversal e analítico, conduzido com 418 idosos com indicativo de depressão residentes no município de Uberaba, MG. Utilizaram-se a Escala de Depressão Geriátrica Abreviada e o Fenótipo de Fragilidade de Fried. Foram utilizados análise descritiva e o teste Qui-quadrado $(p<0,05)$. Resultados: Verificou-se que, entre os idosos com indicativo de depressão, 27,8\% eram frágeis e 51,7\%, pré-frágeis. O status de fragilidade não esteve associado ao sexo $(p=0,910)$. Dentre os pré-frágeis, os componentes do fenótipo mais impactados foram o autorrelato de exaustão/fadiga para as mulheres e diminuição da força muscular para os homens. Nos frágeis, prevaleceu a diminuição da força muscular para ambos os sexos. Conclusão: Mediante os achados deste estudo, conclui-se que, embora não tenha ocorrido associação entre a síndrome de fragilidade e o sexo, a identificação dos componentes do fenótipo de fragilidade mais impactados pode favorecer o atendimento multiprofissional, considerando as especificidades dos grupos. O diagnóstico precoce contribui para o estabelecimento de condutas e prevenção de agravos.

\section{ABSTRACT}

Objectives: To describe the socioeconomic variables of elderly indicative of depression by gender, verify the association between frailty status and sex, and describe the component of the phenotype of frailty among older adults impacted more indicative of pre-frail and frail depression. Methods: An observational, cross-sectional, analytical, conducted with 418 elderly residents indicative of depression in Uberaba-MG. We used the Geriatric Depression Scale Short and Phenotype of Frailty Fried. We used descriptive and Chi-square test $(p<0.05)$ analysis. Results: It was found that among elderly indicative of depression, $27.8 \%$ and $51.7 \%$ were fragile pre-frail. The frailty status was not associated with gender $(p=0.910)$. Among the pre-frail, components most impacted phenotype were self-reported exhaustion/fatigue for women and decreased muscle strength for men. In fragile, prevailed decreased muscle strength for both sexes. Conclusion: Through the findings of this study, it is concluded that

1 Universidade Federal do Triângulo Mineiro (UFTM), Instituto de Ciências da Saúde, Grupo de Pesquisa em Saúde Coletiva.

Recebido em

$$
25 / 6 / 2014
$$

Aprovado em

$10 / 11 / 2014$

DOI: 10.1590/0047-2085000000044
Endereço para correspondência: Darlene Mara dos Santos Tavares

Rua Jonas de Carvalho, 420, Bairro Olinda

38055-440 - Uberaba, MG, Brasil

E-mail: darlenetavares@enfermagem.uftm.edu.br 


\section{Keywords}

Frail elderly, depression, sex

distribution. though there was no association between the syndrome of frailty and sex, the identification of components of the phenotype of frailty most impacted can promote multidisciplinary care, considering the specificities of the groups. Early diagnosis helps to establish conducts and disease prevention.

\section{INTRODUÇÃO}

Atualmente, existem cerca de 350 milhões de pessoas no mundo com depressão', sendo uma das mais frequentes causas de sofrimento emocional na vida adulta e que diminui significativamente a qualidade de vida de idosos ${ }^{2}$. Essa morbidade pode caracterizar-se de modo subsindrômico, o que dificulta o diagnóstico².

Estudos realizados no Brasil, com idosos residentes na comunidade, apontam para prevalência de indicativo de depressão de aproximadamente $21 \% \%^{3,4}$. Destaca-se que essa doença pode tornar-se crônica ou recorrente, interferindo na capacidade de realização de atividades rotineiras e, em situações mais extremas, pode ser fator predisponente para o suicídio'. Assim, tanto por sua prevalência quanto pelas consequências, a depressão representa um importante problema de saúde pública 5 .

Dessa forma, o conhecimento e a identificação de fatores relacionados à depressão entre idosos são relevantes, visto que podem favorecer a elaboração de estratégias e ações precoces por equipes multiprofissionais ${ }^{6}$. Nesse contexto, pesquisadores têm buscado estabelecer a relação entre depressão e a síndrome de fragilidade, outro importante determinante de morbimortalidade em idosos? ${ }^{7}$.

Fragilidade em idosos pode ser definida como uma "síndrome médica com múltiplas causas e fatores contribuintes caracterizada pela diminuição de força, resistência e funções fisiológicas reduzidas que aumentam a vulnerabilidade de um indivíduo para o desenvolvimento de dependência funcional e/ou morrer"s.

Um estudo brasileiro ${ }^{9}$ e um estudo espanhol ${ }^{10}$ destacaram maior percentual de pré-frágeis entre idosos na comunidade, com prevalências de $46 \%$ e 47\%, respectivamente. Pesquisas internacionais também verificaram a associação entre síndrome de fragilidade e depressão ${ }^{10,11}$. Investigação conduzida com idosos na China evidenciou que as condições de pré-fragilidade e fragilidade estiveram associadas ao aumento do número de sintomas depressivos, com maior prevalência entre os frágeis ${ }^{12}$.

Acredita-se que a depressão seja um dos fatores de risco para fragilidade. Características como reclusão e inapetência, típicas do quadro depressivo, poderiam desencadear a perda de massa muscular, força e tolerância ao exercício ${ }^{11}$, itens esses avaliados na síndrome de fragilidade.

Grande parte das pesquisas a respeito de depressão e/ou fragilidade enfatiza mulheres, havendo carência de estudos relacionando o tema ao sexo masculino ${ }^{7,11}$.
Considerando, portanto, o impacto da depressão e da síndrome de fragilidade sobre os idosos e que o processo de envelhecimento pode ser vivenciado de forma diferente entre homens e mulheres, este estudo pretendeu contribuir para ampliação do conhecimento nessa área, de forma a favorecer a elaboração de ações assistenciais que considerem as especificidades desses grupos.

Assim, este estudo teve por objetivos descrever as variáveis socioeconômicas de idosos com indicativo de depressão segundo o sexo, verificar a associação entre o status de fragilidade e o sexo e descrever o componente do fenótipo de fragilidade mais impactado entre os idosos com indicativo de depressão pré-frágeis e frágeis.

\section{MÉTODOS}

Trata-se de um inquérito domiciliar, com abordagem quantitativa, transversal e analítica, desenvolvido com idosos residentes na área urbana do município de Uberaba, MG. Este estudo faz parte de uma pesquisa maior desenvolvida pelo Grupo de Pesquisa em Saúde Coletiva, da Universidade Federal do Triângulo Mineiro (UFTM).

Para composição da amostra, obteve-se uma lista, junto ao Centro de Zoonoses do município, contendo o nome e o endereço completo dos idosos. Para seleção dos idosos, consideraram-se $95 \%$ de confiança, $80 \%$ de poder do teste, margem de erro de 4,0\% para as estimativas intervalares e uma proporção estimada de $\pi=0,5$ para as proporções de interesse. Assim, em 2012 compuseram a amostra 2.118 idosos, sendo entrevistados 1.693 e cancelados 265 por óbito e 160 por declínio cognitivo.

Foram considerados critérios de inclusão para a presente pesquisa: ter 60 anos ou mais de idade, ambos os sexos, não apresentar declínio cognitivo e ter indicativo de depressão. Foram excluídos os idosos que apresentavam incapacidade de deambular sozinhos, sequelas graves de acidente vascular encefálico (AVE), doença de Parkinson em estágio grave ou instável, condição/estágio terminal, déficit grave de visão e/ou audição ( $n=29$ ), aqueles que não responderam a todos os itens de fragilidade $(n=55)$, e sem indicativo de depressão $(n=1.191)$. Portanto, participaram do presente estudo 418 idosos, sendo 120 do sexo masculino e 298 do feminino.

Para a coleta de dados, foram selecionados 10 entrevistadores, os quais foram treinados pelo pesquisador responsável a respeito da forma de abordagem do idoso, aplicação dos questionários e sobre os aspectos éticos da pesquisa. 
As entrevistas foram revisadas por supervisores que verificaram a existência de campos incompletos ou inconsistência nas respostas. Nestes casos, os questionários foram devolvidos aos entrevistadores para o preenchimento adequado.

Foram utilizados os seguintes instrumentos para a coleta de dados:

- Miniexame do Estado Mental (MEEM) para verificar a presença de declínio cognitivo. O MEEM possui escore que varia de 0 a 30 pontos, sendo que os pontos de corte para declínio cognitivo variam em função da escolaridade do idoso: 13 pontos para analfabetos, 18 pontos para escolaridade de 1 a 11 anos e 26 pontos para escolaridade superior a 11 anos $^{13}$.

- Questionário Brasileiro de Avaliação Funcional e Multidimensional (BOMFAQ), adaptado do questionário Older Americans Resources and Services (OARS), empregado previamente em estudos com idosos residentes em São Paulo ${ }^{14}$, para a coleta dos dados sociodemográficos e econômicos.

- Escala de Depressão Geriátrica Abreviada15 para avaliar a presença de indicativo de depressão. Trata-se de uma escala de rastreio, que contém 15 perguntas com respostas objetivas (sim ou não) e escore que varia de 0 a 15 pontos. Considera-se indicativo de depressão quando o idoso apresenta pontuação igual ou superior a cinco pontos ${ }^{15}$.

- Síndrome de fragilidade, avaliada por meio dos cinco componentes do fenótipo de fragilidade, proposto por Fried et al. ${ }^{16}$ : (1) perda de peso não intencional, avaliada por meio da seguinte pergunta: "No último ano, o senhor perdeu mais do que $4,5 \mathrm{~kg}$ ou $5 \%$ do peso corporal sem intenção (isto é, sem dieta ou exercício)?"; (2) autorrelato de exaustão e/ou fadiga, mensurado por meio de duas questões (itens 7 e 20) da versão brasileira da escala de depressão do CES-D ${ }^{17}$. Os idosos que obtiveram escore 2 ou 3 em qualquer uma das questões preencheram o critério de fragilidade para este item ${ }^{16}$; (3) diminuição da força muscular, verificada com base na força de preensão palmar, por meio do dinamômetro hidráulico manual do tipo JAMAR, modelo SAEHAN ${ }^{\circledR}$ SH5001 - 973. Foram obtidas três medidas, apresentadas em quilograma/força (kgf), com um intervalo de um minuto entre elas, sendo considerado o valor médio destas, adotando-se os pontos de corte propostos por Fried et al. ${ }^{16}$; (4) lentidão na velocidade de marcha, em que se considerou o tempo de marcha (em segundos) gasto para percorrer uma distância de 4,6 metros. O idoso percorreu uma distância total de 8,6 metros, sendo os dois metros iniciais e os dois metros finais desconsiderados para o cálculo do tempo gasto na marcha. Foram realizadas três medidas, apresentadas em segundos, considerando-se o valor médio destas. Utilizaram-se como padrão o uso de um cronômetro profissional da marca Vollo ${ }^{\circledR}$, modelo VL-1809, e os pontos de corte propostos por Fried et al. ${ }^{16}$ e (5) baixo nível de atividade física, verificado pela versão longa do Questionário Internacional de Atividade Física (IPAQ), adaptada para idosos $^{18}$. A classificação empregada para esse componente considerou ativos aqueles que dispendiam 150 minutos ou mais de atividade física semanal; e inativos idosos que dispendiam de 0 a 149 minutos de atividade física semanal. Idosos com três ou mais desses itens foram classificados como frágeis e aqueles com um ou dois itens, como pré-frágeis. Aqueles com todos os testes negativos para a síndrome de fragilidade foram considerados não frágeis ${ }^{16}$.

Foram incluídas as variáveis sociodemográficas: sexo (masculino e feminino), faixa etária (60 a 70 anos, 70 a 80 anos, 80 anos ou mais), situação conjugal (casado ou mora com companheiro; separado ou desquitado ou divorciado; viúvo; e solteiro), escolaridade, em anos de estudo (sem escolaridade; 1 a 4; 4 a 8; 8; 9 a 11 e 11 ou mais), e renda individual, em salários-mínimos (sem renda; < 1; 1; 1 a 3; 3 a 5; $>$ 5); presença de indicativo de depressão: sim ou não; classificação da síndrome de fragilidade: não frágil, pré-frágil ou frágil; fenótipo de fragilidade: perda de peso não intencional no último ano, diminuição da força muscular, autorrelato de exaustão e/ou fadiga, lentidão na velocidade da marcha e baixo nível de atividade física.

Após a coleta de dados, foi construído um banco de dados eletrônico, no programa Excel ${ }^{\circledR}$, processados em microcomputador, por duas pessoas, em dupla entrada. Foram verificadas a existência de registros duplicados e a consistência dos campos. Quando houve dados inconsistentes, a entrevista original foi retomada para a correção. Posteriormente, o banco de dados foi importado para o software "Statistical Package for Social Sciences" (SPSS) versão 17.0 para a análise dos dados.

Foi realizada análise estatística descritiva para as variáveis categóricas, a partir de frequências absolutas e percentuais. Para comparação das variáveis, foi aplicado o teste Qui-quadrado, considerando as associações significativas quando $p$ $<0,05$.

O projeto foi submetido ao Comitê de Ética em Pesquisa com Seres Humanos da UFTM e aprovado sob parecer n 2265. Os entrevistadores abordaram os idosos da pesquisa no domicílio, apresentando o Termo de Consentimento Livre e Esclarecido. Depois de esclarecidas as dúvidas dos participantes, foi solicitada a assinatura do referido termo e se deu início à entrevista.

\section{RESULTADOS}

Em ambos os sexos, prevaleceram os idosos na faixa etária de 70 a 80 anos, com 1 a 4 anos de estudo e renda individual 
de um salário-mínimo. Quanto à situação conjugal, a maioria dos homens era casada ou morava com a companheira, enquanto a maioria das mulheres era viúva (Tabela 1).

A tabela 1 apresenta a distribuição das variáveis socioeconômicas dos idosos com indicativo de depressão segundo o sexo.

Tabela 1. Variáveis socioeconômicas de idosos com indicativo de depressão, segundo o sexo. Uberaba-MG, 2012

\begin{tabular}{|c|c|c|c|c|}
\hline \multirow{2}{*}{ Variáveis socioeconômicas } & \multicolumn{2}{|c|}{ Homens } & \multicolumn{2}{|c|}{ Mulheres } \\
\hline & $\mathrm{n}$ & $\%$ & $\mathrm{n}$ & $\%$ \\
\hline \multicolumn{5}{|l|}{ Faixa etária (em anos) } \\
\hline 60 a 70 & 41 & 34,2 & 119 & 39,9 \\
\hline 70 a 80 & 48 & 40 & 125 & 41,9 \\
\hline 80 e mais & 31 & 25,8 & 54 & 18,1 \\
\hline \multicolumn{5}{|l|}{ Estado conjugal } \\
\hline Casado ou mora com o companheiro & 76 & 63,3 & 89 & 29,9 \\
\hline Separado/Desquitado/Divorciado & 13 & 10,8 & 36 & 12,1 \\
\hline Viúvo & 26 & 21,7 & 153 & 51,3 \\
\hline Solteiro & 5 & 4,2 & 20 & 6,7 \\
\hline \multicolumn{5}{|l|}{ Escolaridade (em anos de estudo) } \\
\hline Sem escolaridade & 30 & 25 & 72 & 24,2 \\
\hline 1 a 4 & 56 & 46,7 & 169 & 56,7 \\
\hline 4 a 8 & 13 & 10,8 & 25 & 3,7 \\
\hline 8 & 9 & 7,5 & 11 & 3,7 \\
\hline 9 a 11 & 3 & 2,5 & 4 & 1,3 \\
\hline 11 ou mais & 9 & 7,5 & 17 & 5,7 \\
\hline \multicolumn{5}{|l|}{ Renda individual (salários-mínimos)* } \\
\hline Sem renda & 5 & 4,2 & 34 & 11,4 \\
\hline$<1$ & 2 & 1,7 & 10 & 3,4 \\
\hline 1 & 65 & 54,2 & 175 & 58,7 \\
\hline 1 a 3 & 38 & 31,7 & 67 & 22,5 \\
\hline 3 a 5 salários-mínimos & 6 & 5 & 10 & 3,4 \\
\hline$>5$ & 4 & 3,3 & 2 & 0,7 \\
\hline
\end{tabular}

*Salário-mínimo em 2013: $R \$ \$$ 678,00 reais $^{23}$.

A prevalência da condição de fragilidade em idosos com indicativo de depressão foi de $27,8 \%$, enquanto a pré-fragilidade correspondeu a 51,7\%. Não houve diferença significativa na comparação do status de fragilidade segundo sexo ( $p=$ $0,910)$. A maioria em ambos os grupos era pré-frágil (Tabela 2 ).

A comparação dos status de fragilidade dos idosos segundo sexo está disposta na tabela 2.

Dentre os pré-frágeis, os componentes do fenótipo mais impactados foram o autorrelato de exaustão/fadiga para o sexo feminino $(38,8 \%)$ e diminuição da força muscular para o masculino (45,3\%). Nos frágeis, prevaleceu a diminuição da força muscular para as mulheres (83,3\%) e homens $(90,6 \%)$ (Tabela 3).

A tabela 3 apresenta a distribuição dos idosos pré-frágeis e frágeis quanto aos componentes do fenótipo de fragilidade, segundo sexo.
Tabela 2. Comparação dos status de fragilidade de idosos com indicativo de depressão segundo sexo. Uberaba-MG, 2012

\begin{tabular}{|c|c|c|c|c|c|c|c|c|}
\hline \multirow{2}{*}{ Sexo } & \multicolumn{2}{|c|}{ Homens } & \multicolumn{2}{|c|}{ Mulheres } & \multicolumn{2}{|c|}{ Total } & \multirow{2}{*}{$\chi^{2}$} & \multirow{2}{*}{$p^{*}$} \\
\hline & $n$ & $\%$ & $\mathrm{n}$ & $\%$ & $n$ & $\%$ & & \\
\hline Status de fragilidade & & & & & & & 0,188 & 0,910 \\
\hline Frágil & 32 & 26,7 & 84 & 28,2 & 116 & 27,8 & & \\
\hline Pré-frágil & 64 & 53,3 & 152 & 51 & 216 & 51,7 & & \\
\hline Não frágil & 24 & 20 & 62 & 20,8 & 86 & 20,6 & & \\
\hline
\end{tabular}

Tabela 3. Distribuição dos idosos pré-frágeis e frágeis com indicativo de depressão quanto aos componentes do fenótipo de fragilidade segundo sexo. Uberaba-MG, 2012

\begin{tabular}{|c|c|c|c|c|c|c|c|c|c|}
\hline \multirow{2}{*}{\multicolumn{2}{|c|}{ Fenótipo de fragilidade }} & \multicolumn{4}{|c|}{ Pré-frágeis } & \multicolumn{4}{|c|}{ Frágeis } \\
\hline & & \multicolumn{2}{|c|}{ Homens } & \multicolumn{2}{|c|}{ Mulheres } & \multicolumn{2}{|c|}{ Homens } & \multicolumn{2}{|c|}{ Mulheres } \\
\hline & & $n$ & $\%$ & $n$ & $\%$ & $\mathrm{n}$ & $\%$ & $\mathrm{n}$ & $\%$ \\
\hline \multirow{2}{*}{$\begin{array}{l}\text { Perda de } \\
\text { peso não } \\
\text { intencional }\end{array}$} & Sim & 13 & 20,3 & 44 & 28,9 & 18 & 56,2 & 38 & 45,2 \\
\hline & Não & 51 & 79,7 & 108 & 71,1 & 14 & 43,8 & 46 & 54,8 \\
\hline \multirow{2}{*}{$\begin{array}{l}\text { Autorrelato } \\
\text { de exaustão } \\
\text { e/ou fadiga }\end{array}$} & Sim & 17 & 26,6 & 59 & 38,8 & 19 & 59,4 & 62 & 73,8 \\
\hline & Não & 47 & 73,4 & 93 & 61,2 & 13 & 40,6 & 22 & 26,2 \\
\hline \multirow{2}{*}{$\begin{array}{l}\text { Lentidão na } \\
\text { velocidade } \\
\text { de marcha }\end{array}$} & Sim & 7 & 10,9 & 39 & 25,7 & 19 & 59,4 & 52 & 61,9 \\
\hline & Não & 57 & 89,1 & 113 & 74,3 & 13 & 40,6 & 32 & 38,1 \\
\hline \multirow{2}{*}{$\begin{array}{l}\text { Baixo nível } \\
\text { de atividade } \\
\text { física }\end{array}$} & Sim & 25 & 39,1 & 33 & 21,7 & 25 & 78,1 & 59 & 70,2 \\
\hline & Não & 39 & 60,9 & 119 & 78,3 & 7 & 21,9 & 25 & 29,8 \\
\hline \multirow{2}{*}{$\begin{array}{l}\text { Diminuição } \\
\text { da força } \\
\text { muscular }\end{array}$} & Sim & 29 & 45,3 & 57 & 37,5 & 29 & 90,6 & 70 & 83,3 \\
\hline & Não & 35 & 54,7 & 95 & 62,5 & 3 & 9,4 & 14 & 16,7 \\
\hline
\end{tabular}

\section{DISCUSSÃO}

A faixa etária de 70 a 80 anos é condizente ao estudo com base no Projeto EPIDOSO, no qual prevaleceram aqueles com 70 a 80 anos $^{19}$. Destaca-se que, no presente estudo, esse fato pode estar relacionado à especificidade da amostra, considerando que os idosos têm sido acompanhados pelo Grupo de Pesquisa em Saúde Coletiva desde 2005.

A escolaridade prevalente é divergente da obtida entre idosos no Sudão, sendo o indicativo de depressão mais frequente entre aqueles sem escolaridade (51\%), seguido pelos com grau primário $(42,9 \%)^{20}$. No entanto, destaca-se que no Brasil a maioria dos idosos apresenta até quatro anos de estudo $(50,2 \%)^{21}$ e que a baixa escolaridade pode contribuir para quadros depressivos entre idosos comunitários ${ }^{6}$.

O maior número de homens casados e mulheres viúvas encontrado no presente estudo pode estar relacionado à menor longevidade masculina, contribuindo para que a viuvez entre os homens seja menor em relação às mulheres. Em 2010, a expectativa de vida das mulheres ao nascer era de 
78,3 anos e, para os homens, 71 anos $^{22}$. Porém, destaca-se que ser solteiro, separado ou divorciado constituem-se fatores de risco para quadros depressivos entre idosos ${ }^{6}$.

A prevalência da renda individual de um salário-mínimo é convergente à renda domiciliar per capita do idoso brasileiro $(43,2 \%)^{21}$. Pesquisa de revisão destacou que a condição socioeconômica desfavorável pode ser preditiva de quadros depressivos entre idosos na comunidade ${ }^{\text {. }}$

Salienta-se que os dados socioeconômicos obtidos no presente estudo aproximam-se das características gerais da população idosa no país. Desse modo, não se pode estabelecer uma relação de causalidade entre esses fatores e o indicativo de depressão.

No que se refere à prevalência dos estados de fragilidade na amostra pesquisada, estudo conduzido nos Estados Unidos constatou percentuais similares em idosos com sintomas depressivos para a condição de pré-fragilidade $(52,7 \%)$ e fragilidade $(26,5 \%)^{24}$. Esses resultados corroboram com investigação realizada na Holanda entre idosos comunitários com indicativo de depressão, quanto à prevalência de fragilidade $(27,2 \%)^{25}$.

A fragilidade é considerada um fator de risco para o desenvolvimento e persistência de sintomas depressivos ${ }^{12}$. Em contrapartida, aspectos característicos de indivíduos com sintomatologia depressiva como mudanças de comportamento, atividade e compromisso social podem culminar com o declínio funcional e a fragilidade ${ }^{26}$.

Nesta perspectiva, o aprofundamento e a compreensão dessas relações podem representar implicações para o desenvolvimento de ações e intervenções direcionadas ao campo da saúde pública ${ }^{7}$. A identificação da síndrome de fragilidade em idosos com sintomas depressivos é relevante, uma vez que essa condição é altamente negligenciada nos cuidados de saúde mental ${ }^{25}$. Ademais, o rastreio de idosos com sintomas depressivos, assim como o diagnóstico e o tratamento da depressão, pode se constituir como estratégia na redução do risco de fragilidade ${ }^{26}$.

A ausência de diferença na comparação do status de fragilidade entre o sexo pode ser apoiada nos resultados de um estudo americano, em que o grau de sobreposição na construção do modelo explicativo entre depressão e fragilidade não variou significativamente em função do sexo, apesar de as mulheres terem sido mais propensas à fragilidade e aos sintomas depressivos ${ }^{24}$.

Dentre os componentes do fenótipo de fragilidade, o autorrelato de exaustão e/ou fadiga foi o que apresentou maior percentual entre as idosas pré-frágeis e frágeis. Esse resultado pode estar relacionado às maiores prevalências de fragilidade 24,27 e da manifestação de queixas somáticas decorrentes de quadros depressivos ${ }^{24,28}$, além da provável influência imperativa da realização de tarefas domésticas, em consonância com condições de baixa força muscular ${ }^{29}$. Investigação internacional conduzida com dados provenien- tes de países como Dinamarca, Suécia e Finlândia constatou que a confluência de características específicas de fragilidade, como a fadiga e a lentidão na velocidade de marcha e doença depressiva, esteve associada a um risco aumentado de morte, especialmente em mulheres idosas ${ }^{30}$.

Destaca-se que esse componente fenotípico mantém associação com baixa velocidade de marcha e desempenho físico, mobilidade prejudicada, incapacidade funcional e depressão ${ }^{31}$. Além disso, a síndrome de fragilidade se manifesta com o aumento na expressão de mediadores inflamatórios, que pode contribuir para condições como fraqueza, mal-estar e fadiga ${ }^{32,33}$, aspectos esses característicos de idosos com sintomas depressivos ${ }^{34}$.

Os resultados do presente estudo sugerem que a investigação da fadiga e/ou exaustão, além da compreensão de repercussões advindas dos múltiplos fatores envolvidos nesse componente, pode se constituir como aspecto preponderante para o cuidado direcionado às idosas com sintomas depressivos e/ou em condição de pré-fragilidade e fragilidade.

A diminuição da força muscular mensurada por meio da força de preensão palmar correspondeu ao componente de maior percentual entre os idosos pré-frágeis. Nos frágeis representou maioria, independentemente do sexo. Essa constatação pode ser corroborada por investigação internacional ao apontar que idosos deprimidos apresentaram maiores deficiências na linha de base para os componentes do fenótipo de fragilidade, o que incluiu a força de preensão palmar $^{30}$. Sinalizado como manifestação inicial de fragilidade ${ }^{35}$, esse componente mantém íntima relação com a sarcopenia, um dos elementos constituintes da tríade dessa síndrome ${ }^{16}$. Ademais, destaca-se que idosos depressivos podem apresentar perda de peso, tornarem-se inativos e desencadear decréscimo de massa e força muscular, potencializando o desenvolvimento da síndrome de fragilidade ${ }^{11,34}$.

Acredita-se que o monitoramento e a incorporação de medidas de rastreio e/ou avaliação na prática clínica, assim como programas direcionados à manutenção da força muscular nos diversos níveis de serviços, são salutares para a prevenção e minimização dos efeitos deletérios advindos da síndrome de fragilidade em interface com a sintomatologia depressiva em idosos.

O recorte transversal do presente estudo não permite estabelecer relações de causalidade. Além disso, a escassez de estudos com foco em idosos com indicativo de depressão segundo o sexo dificultou a discussão dos resultados desta pesquisa. Desse modo, sugere-se que novos estudos sejam realizados de modo a constatar a possível relação causal entre síndrome de fragilidade e depressão. Ainda assim, considera-se que o presente estudo permitiu ampliar as discussões sobre a relação entre a síndrome de fragilidade e o indicativo de depressão, considerando as especificidades existentes entre os sexos no processo de envelhecimento. 


\section{CONCLUSÕES}

No presente inquérito, prevaleceram, em ambos os sexos, idosos na faixa etária de 70 a 79 anos e com baixas escolaridade e renda. Verificou-se ainda maior percentual de homens casados e mulheres viúvas. Não houve diferença significativa na comparação do status de fragilidade segundo sexo. A maioria em ambos os grupos era pré-frágil. Dentre os pré-frágeis, os componentes do fenótipo que exerceram maior impacto foram o autorrelato de exaustão/fadiga para o sexo feminino e diminuição da força muscular para o masculino. Nos frágeis, prevaleceu a diminuição da força muscular para mulheres e homens.

Faz-se necessário que os profissionais de saúde ponderem a condição de fragilidade nos cuidados direcionados aos idosos com indicativo de depressão. Nesse sentido, a identificação dessa síndrome geriátrica e seus componentes fenotípicos pode se constituir como estratégia para a tomada de decisões e condutas precoces em equipes multiprofissionais de saúde. Salienta-se, ainda, a necessidade de utilização de critérios diagnósticos para a depressão.

\section{CONTRIBUIÇÕES INDIVIDUAIS}

Darlene Mara dos Santos Tavares - Concepção e desenho do estudo, interpretação dos dados, elaboração do artigo e revisão crítica do seu conteúdo intelectual e aprovação da versão final a ser publicada.

Esthefânia Garcia de Almeida - Concepção e desenho do estudo e interpretação dos dados, contribuição substancial na elaboração do artigo e aprovação da versão final a ser publicada.

Flavia Aparecida Dias - Concepção e desenho do estudo, interpretação e análise dos dados, elaboração do artigo e revisão crítica do seu conteúdo intelectual e aprovação da versão final a ser publicada.

Pollyana Cristina dos Santos Ferreira - Concepção e desenho do estudo, interpretação e análise dos dados, elaboração do artigo e revisão crítica do seu conteúdo intelectual e aprovação da versão final a ser publicada.

Maycon Sousa Pegorari - Concepção e desenho do estudo, interpretação e análise dos dados, elaboração do artigo e revisão crítica do seu conteúdo intelectual e aprovação da versão final a ser publicada.

\section{CONFLITOS DE INTERESSE}

Todos os autores deste manuscrito declaram que não possuem conflitos de interesse.

\section{REFERÊNCIAS}

1. World Health Organization (WHO). Depression: a global crisis. World Mental Health Day. 2012. Disponível em: http://www.who.int/mental_health/management/depression/ wfmh_paper_depression_wmhd_2012.pdf. Acesso em: 10 maio, 2013.

2. Blazer DG. Depression in late life: review and commentary. FOCUS. 2009;7(1):118-36.

3. Hoffmann EJ, Ribeiro F, Farnese JM, Lima EWB. Sintomas depressivos e fatores associados entre idosos residentes em uma comunidade no norte de Minas Gerais, Brasil. J Bras Psiquiatr. 2010;59(3):190-7.

4. Borges DT, Dalmolin BM. Depressão em idosos de uma comunidade assistida pela Estratégia de Saúde da Família em Passo Fundo, RS. Rev Bras Med Fam Comunidade. 2012;7(23):75-82

5. Cunha RV, Bastos GAN, Del Duca GF. Prevalência de depressão e fatores associados em comunidade de baixa renda de Porto Alegre, Rio Grande do Sul. Rev Bras Epidemiol. 2012;15(2):346-54

6. Pinho MX, Custódio 0, Makdisse M. Incidência de depressão e fatores associados em idosos residentes na comunidade: revisão da literatura. Rev Bras Geriatr Gerontol. 2009;12(1):123-40.

7. Mezuk B, Edwards L, Lohman M, Choi M, Lapane K. Depression and frailty in later life: a synthetic review. Int J Geriatr Psychiatry. 2012;27(9):879-92.

8. Morley JE, Vellas B, van Kan GA, Anker SD, Bauer JM, Bernabei R et al. Frailty consensus: a call to action. J Am Med Dir Assoc. 2013;14(6):392-7.

9. Vieira RA, Guerra RO, Giacomin KC, Vasconcelos KSS, Andrade ACS, Pereira LSM, et al. Prevalência de fragilidade e fatores associados em idosos comunitários de Belo Horizonte, Minas Gerais, Brasil: dados do Estudo FIBRA. Cad Saúde Pública. 2013;29(8):1631-43.

10. Jürschik P, Nunin C, Botigué T, Escobar MA, Lavedán A, Viladrosa M. Prevalence of frailty and factors associated with frailty in the elderly population of Lleida, Spain: The FRALLE survey. Arch Gerontol Geriatr. 2012;55(3):625-31.

11. Ní Mhaoláin AM, Fan CW, Romero-Ortuno R, Cogan L, Cunningham C, Kenny RA, et al. Frailty, depression, and anxiety in later life. Int Psychogeriatr. 2012;24(8):1265-74.

12. Feng $L$, Nyunt MS, Feng L, Yap KB, Ng TP. Frailty predicts new and persistent depressive symptoms among community-dwelling older adults: findings from Singapore longitudinal aging study. J Am Med Dir Assoc. 2014;15(1):76.e7-76.e12.

13. Bertolucci PHF, Brucki SMD, Campacci SR, Juliano Y. 0 mini-exame do estado mental em uma populaçäo geral: impacto da escolaridade. Arq Neuro-Psiquiatr. 1994;52(1):1-7.

14. Ramos LR, Toniolo J, Cendoroglo MS, Garcia JT, Najas MS, Perracini M, et al. Two-year follow-up study of elderly residents in S. Paulo, Brazil: methodology and preliminary results. Rev Saúde Publica. 1998;32(5):397-407.

15. Almeida OP, Almeida SA. Confiabilidade da versão brasileira da Escala de Depressão em Geriatria (GDS) versão reduzida. Arq Neuro-Psiquiatr. 1999;57(2B):421-6.

16. Fried LP, Tangen CM, Walston J, Newman AB, Hirsch C, Gottdiener J, et al. Frailty in older adults: evidence for a phenotype. J Gerontol A Med Sci Biol Sci. 2001;56(3):M146-56.

17. Batistoni SST, Neri AL, Cupertino APFB. Validade da escala de depressão do Center for Epidemiological Studies entre idosos brasileiros. Rev Saúde Pública. 2007;41(4):598-605.

18. Mazo GH, Benedetti TRB. Adaptação do questionário internacional de atividade física para idosos. Rev Bras Cineantropom Desempenho Hum. 2010;12(6):480-4.

19. Lima MTR, Silva RS, Ramos LR. Fatores associados à sintomatologia depressiva numa coorte urbana de idosos. J Bras Psiquiatr. 2009;58(1):1-7. Disponível em: http://www. scielo.br/scielo.php?pid=S0047-20852009000100001\&script=sci_arttext. Acesso em: 16 maio, 2014.

20. Assil SM, Ziedan ZA. Prevalence of depression and associated factors among elderly Sudanese: a household survey in Khartoum State. East Mediterr Health J. 2013;19(5):435-40.

21. Instituto Brasileiro de Geografia e Estatística (IBGE). Indicadores Sociais Municipais. Uma análise dos resultados do universo do censo demográfico 2010. IBGE, Rio de Janeiro, 2011. Disponível em: http://biblioteca.ibge.gov.br/visualizacao/livros/liv54598.pdf. Acesso em: $20 \mathrm{jul}, 2013$.

22. Instituto Brasileiro de Geografia e Estatística (IBGE). Tábua Completa de Mortalidade. Brasil. 2012. Disponível em: http://www.ibge.gov.br/home/estatistica/populacao/tabuadevida/2012/defaulttab_pdf.shtm. Acesso em: 13 maio, 2014. 
23. Departamento Intersindical de Estatística e Estudos Socioeconômicos (DIEESE). Cesta básica nacional: salário mínimo nominal e necessário. Disponível em: http://www.dieese. org.br/analisecestabasica/salarioMinimo.htm|\#2013. Acesso em: 10 janeiro, 2014.

24. Lohman M, Dumenci L, Mezuk B. Sex differences in the construct overlap of frailty and depression: evidence from the health and retirement study. J Am Geriatr Soc. 2014;62(3):500-5.

25. Collard RM, Comijs HC, Naarding P, Oude Voshaar RC. Physical frailty: vulnerability of patients suffering from late-life depression. Aging Ment Health. 2014;18(5):570-8.

26. John PDS, Tyas SL, Montgomery PR. Depressive symptoms and frailty. Int J Geriatr Psychiatry. 2013;28(6):607-14.

27. Collard RM, Boter H, Schoevers RA, Oude Voshaar RC. Prevalence of frailty in communitydwelling older persons: a systematic review. J Am Geriatr Soc. 2012;60(8):1487-92.

28. Silverstein B, Edwards T, Gamma A, Ajdacic-Gross V, Rossler W, Angst J. The role played by depression associated with somatic symptomatology in accounting for the gender difference in the prevalence of depression. Soc Psychiatry Psychiatr Epidemiol. 2013;48(2):257-63.

29. Costa TB, Neri AL. Medidas de atividade física e fragilidade em idosos: dados do FIBRA Campinas, São Paulo, Brasil. Cad Saúde Pública. 2011;27(8):1537-50.
30. Brown PJ, Roose SP, Fieo R, Liu X, Rantanen T, Sneed JR. Frailty and depression in older adults: a high-risk clinical population. Am J Geriatr Psychiatry. 2013;13:S1064-7481.

31. Morley JE. Developing novel therapeutic approaches to frailty. Curr Pharm Des. 2009;15(29):3384-95.

32. Bilotta C, Bowling A, Casè A, Nicolini P, Mauri S, Castelli M, et al. Dimensions and correlates of quality of life according to frailty status: a cross-sectional study on community-dwelling older adults referred to an outpatient geriatric service in Italy. Health Qual Life Outcomes. 2010;8(56):1-10.

33. Chang SS, Weiss CO, Xue QL, Fried LP. Patterns of comorbid inflammatory diseases in frail older women: the Women's Health and Aging Studies I and II. J Gerontol A Biol Sci Med Sci. 2010;65(4):407-13.

34. Espinoza SE, Fried LP. Risk factors for frailty in the older adult. Clinical Geriatrics. 2007:15(6):37-44.

35. Xue QL, Bandeen-Roche K, Varadhan R, Zhou J, Fried LP. Initial manifestations of frailty criteria and the development of frailty phenotype in the Women's Health and Aging Study II. J Gerontol A Biol Sci Med Sci. 2008;63(9):984-90. 